\begin{tabular}{ll} 
O P E R A T I O N S R E S E A R C H A N D D E C I S I O N S \\
\hline No. 2
\end{tabular}

DOI: $10.37190 /$ ord 210207

\title{
DEFINING A STORAGE-ASSIGNMENT STRATEGY FOR PRECEDENCE-CONSTRAINED ORDER PICKING
}

\author{
MARIA A. M. TRINDAdE ${ }^{1,2^{*}}$, PAUlo S. A. SOUSA ${ }^{1}$, MARIA R. A. MOREIRA ${ }^{1,3}$ \\ ${ }^{1}$ Faculty of Economics, University of Porto, Porto, Portugal \\ ${ }^{2}$ Católica Porto Business School, Católica University, Porto, Portugal \\ ${ }^{3}$ INESC TEC - Science and Technology, Computers and Systems Engineering Institute, Porto, Portugal
}

\begin{abstract}
A zero-one quadratic assignment model has been proposed for dealing with the storage location assignment problem when there are weight constraints. Our analysis shows that operations can be improved using our model. When comparing the strategy currently used in a real-life company with the designed model, we found that the new placement of products allowed a reduction of up to $22 \%$ on the picking distance. This saving is higher than that achieved with the creation of density zones, a procedure commonly used to deal with weight constraints, according to the literature.
\end{abstract}

Keywords: storage location assignment problem (SLAP), correlated policy, precedence constraints

\section{Introduction}

Retail and wholesale are major sectors of the European economy. Together, they generate $11 \%$ of the Europeans' GDP. A third of European companies belong to the retail or wholesale sectors. They are one of the few sectors that are steadily creating employment across Europe [9]. In the last decade, there has been a growing adoption of e-commerce in these sectors. Published sources indicate that $21.8 \%$ of the world's population buys online and that online sales could reach $\$ 4.8$ trillion by 2021 [17]. This forces companies to be smarter about how they use their warehouses and distribution centres. In the retail and wholesale sectors, order-picking processes are still characterised by a high share of manual human work. Currently, the worldwide share of automated warehouses is just 5\% [7]. This low rate is mainly due to the difficulty in replicating human flexibility and motor skills in machines. As a result, order-picking

*Corresponding author, email address: matrindade@porto.ucp.pt

Received 3 September 2020, Accepted 7 May 2021 
operations represent more than $55 \%$ of the picking costs of companies and improving efficiency in this area is key for those companies [13, 14].

The efficiency of order-picking can be improved in several ways, including assigning products to appropriate storage locations (storage strategy) and determining the appropriate route and picking orders in batches (batching) [25]. We focus on storage strategy. Recently, research on the storage location assignment problem (SLAP) has started to consider characteristics of real-world warehouse activities such as product characteristics, human factors, and precedence constraints. This paper deals with SLAP with weight constraints. We investigate the influence of a new storage assignment policy on order-picking efficiency. This paper is inspired by a real-world Portuguese food retailer warehouse. We propose a zeroone quadratic assignment model for manual systems warehouses that operate in a stock environment. The contributions of this paper are threefold.

First, we introduce a rich variation of SLAP with weight constraints inspired by the retail industry. Although SLAP has been well researched in the literature, SLAP subject to constraints still needs further consideration [5]. Recently, this subject has been defined as a gap in the literature by $[25,28]$ and a matter of interest by $[1,22]$.

Second, in existing studies, we found that the solutions to weight constraints were creating density zones $[5,7]$ and/or limiting the maximum weight per pallet $[6,24]$. These strategies might be inefficient for warehouses with a high number of non-uniform products. With density zones, products are placed according to weight and, therefore, the more variations there are in the weight of fast-moving products, the more zones the picker has to visit (that is, the order-picking distance is increased). Furthermore, the capacity constraint is perceived to be insufficient to ensure the physical integrity of the products. For these reasons, this study proposes a new model to deal with the problem at hand. First, we develop an index to measure the similarity of two products in terms of weight, and then we incorporate that index in a well-known storage-assignment model suitable for our case.

Third, this study makes available a new technique, which is easy to understand, and which can easily be implemented in practice. In addition, it may be used to improve the performance of many other retail companies.

This paper is organised as follows. First, we present a brief literature review on the topic. Then, we provide the main methodology employed in this study. Subsequently, we detail the case study that inspired this paper, and we present the main results. Finally, we give the main conclusion and some ideas for future research.

\section{Literature review}

As the focus of this project is on SLAP, we provide a summary of the relevant literature. SLAP concerns the allocation of products into storage locations, to maximise order-picking efficiency. This problem is impacted in two main ways, namely: storage capacity and the physical characteristics of products [20]. 
Several storage policies may be used to allocate products in warehouses. The most recognised of these are: random, dedicated, and class-based. In a random storage policy, products are randomly allocated into the available spaces $[18,23]$. In a dedicated storage policy, products are allocated to fixed locations [6]. In a class-based storage policy, products are grouped into categories, and inside each category, products are randomly allocated. The storage area (based on the accessibility of storage locations) is divided into classes, too [19]. Within the class-based storage method, the correlated storage system may also be used. This policy is based on the idea that products with high values of correlation must be stored close to each other [10].

A lot of studies have been designed to the correlated storage policy. These studies propose various clustering techniques $[3,15,16,21]$ and (meta-)heuristic approaches $[4,15,26]$. However, the studies have often neglected constraints arising in real-world applications. Order-picking is often subject to constraints. These constraints include requiring certain products to be collected before other products, owing to fragility, shape, size, and preferred unloading sequence [5]. Focusing on weight constraints, we found two different approaches that are usually taken into consideration: the creation of density zones, and maximum capacity.

The creation of density zones is where products are allocated into areas according to their weight [5], for example, propose two mathematical models and develop five heuristic methods to solve the order-picking problem with weight, fragility, and category constraints. In this study, products are classified and allocated to a density zone based on their weight. Diaz [8] develops a two-step heuristic procedure of, first, creating density zones and, second, allocating products within those zones by using the model developed by [15].

The maximum capacity approach is where the maximum capacity of a pallet, or the number of boxes that can be loaded on top of each other, is limited, based on weight. Glock and Grosse [12], for example, describe the order-picking system of a U-shape warehouse in a formal model, to examine the impact of different storage assignment policies. In the model, they consider the maximum capacity that a batch can carry. Grosse et al. [13] propose a simulated annealing approach for solving the joint problem of order-batching and order-picking routing. They take into consideration the maximum capacity of a batch. Xiao and Zeng [27] design a correlated storage assignment system with demand dependences to minimise the order-picking distance. The authors also take into consideration the maximum capacity of a batch. A study has been published that deals with the weight precedent constraint by proposing a new picker-routing [28].

\section{Method}

First, we provide the problem description, then the model building concepts and, finally, the model formulation. Given the complexity of the problem at hand and the 
similarity between our problem and that discussed elsewhere [15], we base our allocation model on his design, and we further develop it to incorporate the weight. We also develop and test a program in $\mathrm{C}++$ to calculate the exact distance travelled by the picker, using the locations given by the designed allocation model.

The problem at hand can be defined as stated below:

Given:

- a set of available slots and a set of products to allocate to those same slots,

- the distance from the pick-up/drop-off (PD) point to each one of the slots,

- the distance from one slot to another,

- the warehouse storage capacity expressed in slots,

- the products' co-purchase pattern,

- the products' demand, in a regular month,

- the products' weight.

Determine:

- the assignment of the products within the warehouse's available spaces.

The goal is to minimise the total distance travelled by the picker.

Many SLAP studies assume that the warehouse configuration may be modified to accommodate the products. In this study, however, the warehouse configuration, as well as the routing policy (one-way S-shape routing), are assumed to be fixed. This is due to the fixed layout and narrow aisles of our case company.

We focus, instead, on the improvement of the storage assignment policy. We propose the allocation of products based on three criteria: co-purchase pattern, demand, and weight. The co-purchase pattern is given by the probability of two products appearing together in orders. The higher the probability, the higher the similarity between the two products. The demand is given by the likelihood of one product appearing on orders. The weight criterion is given by the similarity of two products, in terms of weight. In the end, the highest requested products must be placed in the slots that are next to the PD point; the products with higher similarity (in terms of co-purchase pattern) must be placed next to each other; and the products of similar weight must be in slots near each other, to avoid risking the physical integrity of the products.

The procedure is as follows. First, as in [15], this work formulates the problem as a zero-one quadratic assignment model that gives the location of the products by using the products' co-purchase pattern, demand, and weight; the relative distance between the slots; and the relative distance to the PD point. Then, this work calculates the exact distance travelled by the picker through a program design in $\mathrm{C}++$ language for this purpose, using the locations given by the allocation model. The assignment model uses the set of indexes, parameters, and decision variables as below.

Indices:

$i$-product $i$ ( $k$ is also an index for products), $i=1, \ldots, A$,

$j-\operatorname{slot} j$ ( $l$ is also an index for the slots), $j=1, \ldots, P$. 


\section{Parameters:}

$d_{j l}-$ travel distance between slot $j$ and slot $l$,

$f_{i}$ - frequency with which product $i$ appears on the orders $-f_{i}$ is given by the number of orders in which product $i$ appears $\left(o_{i}\right)$ divided by the total number of orders $(O)$

$$
f_{i}=\frac{o_{i}}{O}
$$

$A$ - number of products to be allocated,

$P$ - number of existing slots,

$n_{i}-$ number of slots needed for the product $i$,

$r_{j}$ - relative distance from the PD point to slot $j$,

$y s_{i k}$ - co-purchase pattern of products $i$ and $k,-y s_{i k}$ is inspired in the market basket analysis association rules, and it is given by the number of orders in which product $i$ and $k$ appear together $\left(n_{i k}\right)$ divided by the total number of orders $(N)$

$$
y s_{i k}=\frac{n_{i k}}{N}
$$

$y w_{i k}$ - similarity between products $i$ and $k$, in terms of weight $y w_{i k}$ is given by the relative difference between the weight of product $i\left(w_{i}\right)$ and the weight of product $k\left(w_{k}\right)$

$$
y w_{i k}=1-\frac{\left|w_{i}-w_{k}\right|}{\max \left(\left|w_{i}\right|,\left|w_{k}\right|\right)}
$$

Decision variable:

$x_{i j}$ - (a binary variable) with 1 if the product $i$ is assigned to slot $j$, and 0 otherwise.

Given the indices, parameters, and decision variables presented, the generic model design is formulated as follows:

$$
\min \frac{1}{2} \sum_{i=1}^{A} \sum_{j=1}^{P} \sum_{k=1}^{A} \sum_{l=1}^{P} f_{i} y s_{i k} y w_{i k} d_{j l} x_{i j} x_{k l}+\sum_{i=1}^{A} \sum_{j=1}^{P} f_{i} r_{j} x_{i j}
$$

Subject to:

$$
\begin{gathered}
\sum_{i=1}^{A} x_{i j}=1, \forall j=1, \ldots, P \\
\sum_{j=1}^{P} x_{i j}=n_{i}, \forall i=1, \ldots, A \\
x_{i j}=0,1, \forall i=1, \ldots, A, \forall j=1, \ldots, P
\end{gathered}
$$


where

$$
\sum_{i=1}^{A} n_{i} \leq P
$$

Equation (4) represents our storage assignment model. The first part, given byproduct of $\mathrm{f}_{i}$ (the likelihood that an operator picks product $i$ for an order) and $y s_{i k} y w_{i k} d_{j l}$ aims to reduce the distance covered by the picker within the slots and assign products with similar weight and co-purchase patterns close to each other, simultaneously. The second part of the equation, given by the product of $f_{i}$ and $r_{j}$, defines the expected distance required to travel from the PD point to slot $j$. It is assumed that a picker can travel from slot $j$ to slot $l$. Equation (5) guarantees that only one product $i$ is assigned to slot $j$. Equation (6) assures that the number of slots assigned to product $i$ equals $n_{i}$. Equation (7) constricts the binary variable values to zero or one. Finally, Equation (8) ensures that the number of slots needed by the product does not exceed the number of available slots.

Note that the objective function proposed uses approximated probabilities instead of exact values. Nevertheless, probabilities were also used in other successful approaches such as [8]; therefore, although it is an approximation, it is still suitable for practical use.

The typical size of the problem in this study might involve 375 products and 540 slots, leading to a formulation having about 75937500 binary variables and 915 structural constraints. Thus, the problem is solved for a restricted number of orders.

\section{Case study}

This paper is inspired by a real-life case of a manual warehouse for a company that supplies retail products to over 191 stores, in Northern Portugal. We focus on the non-food stock section of the non-perishables warehouse. The current layout consists of a closed area, divided into several aisles that are assigned to different categories of products such as car tools, lights, cooking equipment and so on. Pickers perform a conventional manual picking operation within a one-way S-shape route. The company has a picker-to-parts system with low-level picking. During the process, the pickers are guided by a voice speaking system that tells them the location of the products. The picking guide is generated by the company management system, which already divides the orders according to the typology (food, non-food and drinks). There are pickers exclusively allocated to the non-food area. Figure 1 shows the warehouse under study. On the lower side of the figure, the docks used to receive and ship the products are represented. 


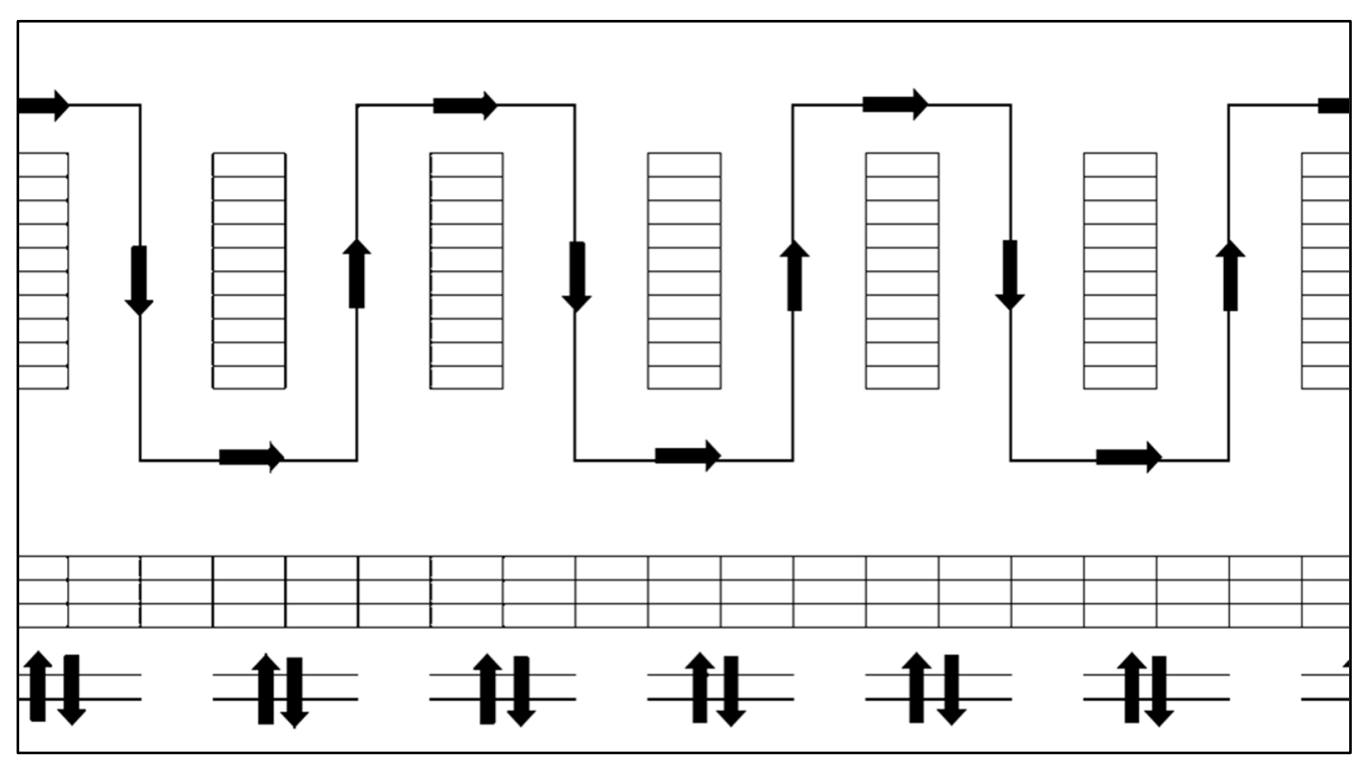

Fig. 1. Warehouse layout scheme adapted from the company report

\section{Results}

This section covers the application of different assignment methods for our case study. SLAP is proved to be a non-deterministic polynomial-time hard (NP-hard) problem [11]. The case company has, on average, 11033 orders per day and up to 400 products per order. For this reason, the optimal solution cannot be obtained for large solution spaces. Thus, the problem is solved by using as a sample the orders of one store in a regular month. We test two scenarios:

- Diaz's method (density zones strategy): first, we distribute products into density zones, according to weight. Then, within those zones, we sort products according to the demand and similarity criteria (following [15]), see [8].

- A new model (scenario designed for this paper): we allocate products based on the combination of co-purchase pattern, demand, and weight criteria, following the model presented.

For the computation of the results, we run the model in DOcplexcloud ${ }^{1}-$ a cloud $^{2}$ with a 10-core processor and $60 \mathrm{~Gb}$ RAM. The model was developed at ILOG Cplex Optimisation Studio 12.9. The stop time was $3600 \mathrm{~s}$. The calculation of the exact orderpicking distance was performed at Visual Studio 15.9 (C++ language). 
Table 1 is an extract of the results obtained in the three scenarios. When comparing the current strategy of the company with the new model, it is found that the new placement of products allows a reduction in the picking distance of up to $22 \%$.

Table 1. Results obtained in the three scenarios in terms of the distance travelled by the picker

\begin{tabular}{|c|c|c|c|}
\hline \multicolumn{1}{|c|}{ Subject } & Diaz's method & New model & Current situation \\
\hline Distance $(\mathrm{km} / \mathrm{month})$ & 24.40 & 19.68 & 25.50 \\
\hline Per cent of improvement & 4.31 & 22.82 & - \\
\hline
\end{tabular}

The generic travelled distance $(\mathrm{km} / \mathrm{month})$ can be converted at a cost $(€ /$ month), quantifying the necessary number of pickers in the system. Table 2 shows the potential savings in each of the scenarios (in comparison to the current scenario of the case company). The allocation of the products in the new model scenario enables a reduction of the distance travelled monthly of $6 \mathrm{~km}$. As the warehouse operates 26 days a month and the picking machines used in the warehouse move at an average speed of $2 \mathrm{~km}$ per hour, operations can be reduced up to 0.22 hours a day. This reduction leads to the conclusion that it is possible to maintain the same warehouse activity level fulfilling the orders of one store, with 0.02 employees less (if each employee works on average 7.5 hours per day). Extrapolating this data for the 191 stores, within a 95\% confidence interval, the potential reduction of pickers goes up to 2 (down limit: 2.83 | upper limit: 2.90).

Table 2. Savings obtained in the different scenarios in terms of the potential reduction of the number of pickers

\begin{tabular}{|l|c|c|}
\hline \multicolumn{1}{|c|}{ Savings } & Diaz's method & New model \\
\hline Distance reduction, km/month & 1.10 & 5.82 \\
\hline Distance reduction, km/day & 0.04 & 0.22 \\
\hline Reduction in daily hours of operation, $\mathrm{h}$ & 0.02 & 0.11 \\
\hline Potential reduction of the number of pickers) & 0.003 & 0.015 \\
\hline Potential reduction of pickers $-\mathrm{CI}_{0.01,95 \%}$ & $0.49-0.65$ & $2.83-2.90$ \\
\hline
\end{tabular}

Note that the implementation of the layout required for each of the scenarios might create costs arising from the changes in the location of the products and the warehouse management system used by the company. The employees would also have to adapt to a different work environment. In addition, these results refer to the specific case study to which the proposed approach is applied. They should not be generalised.

\section{Experimental design}

In this section, we present four experimental designs that allow us to test different ways of calculating the similarity of two products in terms of weight - weight parameter 
(experiments I and II), and to test an alternative way of integrating the weight of products in the model (experiment III). We also run the model for five additional samples (experiment IV) to test the robustness of the procedure.

\subsection{Experiments I and II}

In experiments I and II, we test the use of alternative weight parameters (parameters based on other relative difference indexes) in the model. The difference between experiments I and II is that the relative indexes presented in experiment II do not return values from 0 to 1 (values in line with the other parameters in the model). So, in experiment II, we normalise the indexes. In the next subsections, we provide more details.

In Experiment I, we perform a $3 \times 2$ experimental design, where we test the use of the non-normalised weight parameter $y w_{i k}$ in three ways $\left(y w_{i k}, y w_{i k}^{2}\right.$, and $\left.\sqrt{y w_{i k}}\right)$ for two different indexes. These indexes already return a number between 0 and 1 ; the number in line with the values of the demand and co-purchase pattern parameters. Results are presented in Table 3. The cases in which it is not possible to get a solution are marked with a sign - .

Table 3. Experiment I. Alternative weight parameters (non-normalised indexes)

\begin{tabular}{|c|c|c|c|}
\hline \multirow{2}{*}{ Relative difference indexes } & \multicolumn{3}{|c|}{$\begin{array}{c}\text { Distance } \\
{[\mathrm{km} / \mathrm{month}]}\end{array}$} \\
\cline { 2 - 4 } & $y w_{i k}$ & $\sqrt{y w_{i k}}$ & $y w_{i k}^{2}$ \\
\hline$y w_{i k}=1-\frac{\left|w_{i}-w_{k}\right|}{\frac{w_{i}+w_{k}}{2}}$ & 19.68 & 19.68 & - \\
\hline$y w_{i k}=1-\frac{\left|w_{i}-w_{k}\right|}{\max \left(w_{i} w_{k}\right)}$ & 19.68 & 19.68 & - \\
\hline
\end{tabular}

In Experiment II, we perform a $3 \times 7$ experimental design, where we examine the use of a normalised weight parameter $y w_{i k}^{*}$ in three ways $\left(y w_{i k}^{*}, y w_{i k}^{2^{*}}\right.$, and $\left.\sqrt{y w_{i k}^{*}}\right)$ for seven indexes. In this experiment, all the relative difference indexes are normalised through min-max algorithm to ensure that the index returns a value between 0 and 1 . Results are presented in Table 4. Situations in which it is not possible to get a solution are marked with a sign - . 
Table 4. Experiment II. Alternative weight parameters (normalised indexes)

\begin{tabular}{|c|c|c|c|}
\hline \multirow{2}{*}{ Relative difference indexes } & \multicolumn{3}{|c|}{$\begin{array}{c}\text { Distance } \\
{[\mathrm{km} / \mathrm{mon} \text { th] }}\end{array}$} \\
\cline { 2 - 4 } & $y w_{i k}^{*}$ & $\sqrt{y w_{i k}^{*}}$ & $y w_{i k}^{2^{*}}$ \\
\hline$y w_{i k}=1-\frac{\left|w_{i}-w_{k}\right|}{w_{k}}$ & 19.68 & 19.68 & 19.68 \\
\hline$y w_{i k}=1-\frac{\left|w_{i}-w_{k}\right|}{\frac{w_{i}+w_{k}}{2}}$ & 19.68 & 19.68 & - \\
\hline$y w_{i k}=1-\frac{\left|w_{i}-w_{k}\right|}{\sqrt{w_{k} w_{i}}}$ & 19.68 & 19.68 & 19.68 \\
\hline$y w_{i k}=1-\frac{\left|w_{i}-w_{k}\right|}{\left(\frac{w_{i}^{-1}+w_{k}^{-1}}{2}\right)}$ & 19.68 & 19.68 & 19.68 \\
\hline$y w_{i k}=1-\frac{\left|w_{i}-w_{k}\right|}{\min \left(w_{i}, w_{k}\right)}$ & 19.68 & 19.68 & 19.68 \\
\hline$y w_{i k}=1-\frac{\left|w_{i}-w_{k}\right|}{\max \left(w_{i}, w_{k}\right)}$ & 19.68 & 19.68 & - \\
\hline$y w_{i k}=1-\ln \frac{w_{i}}{w_{k}}$ & 19.68 & 19.68 & 19.68 \\
\hline
\end{tabular}

\subsection{Experiment III}

In experiment III, we perform a $3 \times 2$ experimental design, where we assess an alternative approach that includes the introduction of the normalised weight of a product $w_{i}^{*}$ rather than the similarity of two products in terms of weight $y w_{i k}$, in the zero-one quadratic assignment model (eq. (4)). The weight is normalised through min-max algorithm to ensure that it returns a value between 0 and 1 (eq. (9)) - value that is in line with the other parameters in the model eq. (4).

$$
w_{i}^{*}=1-\frac{w_{i}-w_{i \min }}{w_{i \max }-w_{i \min }}
$$

where $w_{i \min }=\min \left(w_{\mathrm{i}}, \ldots, w_{k}\right)$ and $w_{i \max }=\max \left(w_{i}, \ldots, w_{k}\right), i=1, \ldots, A$.

We test the solution for $w_{i}, w_{i}^{2}, \sqrt{w_{i}}$. Results are presented in Table 5 . 
Table 5. Experiment III. Alternative approach for the objective function (using normalised weight instead of weight similarity)

\begin{tabular}{|c|c|c|c|}
\hline \multirow{2}{*}{ Equation (4) } & \multicolumn{3}{|c|}{$\begin{array}{c}\text { Distance } \\
{[\mathrm{km} / \mathrm{month}]}\end{array}$} \\
\cline { 2 - 4 } & $w_{i}^{*}$ & $w_{i}^{2^{*}}$ & $\sqrt{w_{i}^{*}}$ \\
\hline $\min \frac{1}{2} \sum_{i=1}^{K} \sum_{j=1}^{P} \sum_{k=1}^{K} \sum_{l=1}^{P} f_{i} y s_{i k} d_{j l} x_{i j} x_{k l}+\sum_{i=1}^{K} \sum_{j=1}^{P} f_{i} w_{i} r s_{j} x_{i j}$ & 19.63 & 19.63 & 19.63 \\
\hline
\end{tabular}

In this experiment, we also assess the simultaneous use $w_{i}$ and $w_{i k}$. For this purpose, we use the initial $w_{i k}$ (eq. (3)). We test the solution for $w_{i}, w_{i}^{2}, \sqrt{w_{i}}$. Results are presented in Table 6.

Table 6. Experiment III. Alternative approach for the objective function (using both weight and weight similarity parameters)

\begin{tabular}{|c|c|c|c|}
\hline \multirow{2}{*}{ Equation (4) } & \multicolumn{3}{|c|}{$\begin{array}{c}\text { Distance } \\
{[\mathrm{km} / \mathrm{month}]}\end{array}$} \\
\cline { 2 - 4 } & $w_{i}^{*}$ & $w_{i}^{2^{*}}$ & $\sqrt{w_{i}^{*}}$ \\
\hline $\min \frac{1}{2} \sum_{i=1}^{K} \sum_{j=1}^{P} \sum_{k=1}^{K} \sum_{l=1}^{P} f_{i} y s_{i k} d_{j l} x_{i j} x_{k l}+\sum_{i=1}^{K} \sum_{j=1}^{P} f_{i} w_{i} r s_{j} x_{i j}$ & 19.68 & 19.68 & 19.68 \\
\hline
\end{tabular}

The use of different ways of calculating the weight parameter does not impact the solution. The percentage of improvement is the same in all the alternatives explored in Experiments I and II. Also, the use of the normalised weight of the product (eq. (9)) shows a small improvement in the distance travelled - when compared to the use of the similarity of two products in terms of weight. However, this improvement is not significant (equivalent to $0.2 \%$ ).

\subsection{Experiment IV}

In addition to the three experiments, in Experiment IV, we run the model for the samples of three more stores to see if there are significant differences between the

Table 7. Results obtained with different samples in terms of the distance travelled by the picker

\begin{tabular}{|l|c|c|c|c|c|}
\hline \multicolumn{1}{|c|}{ Components } & Store 1 & Store 2 & Store 3 & Store 4 & Random \\
\hline Distance, new model, km/month & 19.68 & 6.28 & 8.74 & 3.63 & 19.68 \\
\hline Distance, current situation, km/month & 25.50 & 7.98 & 12.07 & 5.04 & 25.98 \\
\hline Per cent of improvement & 22.82 & 23.35 & 27.59 & 27.98 & 24.25 \\
\hline
\end{tabular}


percentages of improvement achieved. We also test the procedure on a random sample in which the frequency is generated from a Gaussian distribution with atmospheric noise (Table 7). The percentages of improvement are even higher in the samples of the other stores. Results indicate that the overall saving could go up to $28 \%$.

\section{Theoretical and managerial implications}

This section highlights the implications of the present study for theory as well as for practice. First, theoretically, a new model is purposed for dealing with SLAP where there are weight constraints. The developed model is of potential interest for warehouses that store a high percentage of non-uniform products and that want to avoid sorting strategies. Second, on the empirical side, the results show that the proposed model is effective in improving overall warehouse operating efficiency. Third, the developed model can potentially help operational managers, in different industries, in the development of a storage assignment policy, allowing them to save time and operate in a faster way. Also, the new model allows the location of items within the aisle to be changed without damaging the results. The model can be further extended to allow the allocation of new products that were not initially considered.

\section{Conclusion}

This paper is inspired by a real-life case of a manual order-picking retail warehouse, with a high number of non-uniform products, where the product weight influences the sequence of order-picking operations. In the literature, real-world constraints are often neglected. This study further develops the model developed by [15] to integrate the weight in the allocation system of the products.

In a numerical study, we compare our model to the current strategy applied by the company under study. Our findings show that the new placement of products allows a reduction of up to $22 \%$ on the picking distance. This percentage is $18 \%$ higher than that achieved using density zones, an approach commonly employed to address the storage location assignment problem.

Our analysis shows that the designed model improves current operations in several respects. Warehouse managers can avoid the strict strategy of sorting products by weight, and they can reduce the picker travel distance for completing customer orders.

The main limitations of the study are the constraints brought by the narrow-aisle layout of the warehouse (namely the application of alternative routing methods, batching operations, and pallet construction processes), and the consideration of one regular 
month as a reference. Also, the model processing time was limited to 3600 (due to software plan constraints).

Future studies can further investigate the effects of using the new model. This could include applying it in different kinds of warehouses, such as those with different picking methods and/or layouts (for example, warehouses with a U-shape or fishbone configuration). There is also the potential to incorporate in the method a different routing and/or batching method. Furthermore, it is possible to include a model of the classification of products to investigate its impact on productivity. Another suggestion is to apply the model to different kinds of companies to research the obtained results and to test the different ways of calculating the co-purchase pattern (i.e., by applying the Jaccard Index, or other purposed similarity indexes e (see for instance, [2]).

\section{Acknowledgements}

We gratefully acknowledge the assistance of the blinded reviewers, who reviewed the manuscript. They helped to improve the quality of the paper.

\section{References}

[1] Akin Bas S., Ahlatcioglu OzKoK B., A fuzzy approach to multi-objective mixed-integer linear programming model for multi-echelon closed-loop supply chain with multi-product multi-time period, Oper. Res. Dec., 2020, 30 (1), 25-46.

[2] Bindi F., Manzini R., PAReschi A., Regattieri A., Similarity coefficients, clustering techniques for the correlated assignment problem in warehousing systems, Proc. 19th International Conference on Production Research, 2007.

[3] Bindi F., MAnzini R., PAReschi A., Regattieri A., Similarity-based storage allocation rules in an order picking system: An application to the food service industry, Int. J. Log. Res., Appl., 2009, 12 (4), 233-247.

[4] BRYNZÉR H., JohANSSON M.I., Storage location assignment. Using the product structure to reduce order picking times, Int. J. Prod. Econ., 1996, 46-47 (1996), 595-603.

[5] Chabot T., Lahyani R., Coelho L.C., Renaud J., Order picking problems under weight, fragility, category constraints, Int. J. Prod. Res., 2017, 55 (21), 6361-6379.

[6] De Koster R., Le-Duc T., Roodbergen K.J., Design, control of warehouse order picking. A literature review, Eur. J. Oper. Res., 2007, 182 (2), 481-501.

[7] DHL, Robotics in Logistics, Retrieved from: https://www.logistics.dhl/global-en/home/insights-andinnovation/thought-leadership/trend-reports/robotics-in-logistics.html, 2018.

[8] DiAz R., Using dynamic demand information, zoning for the storage of non-uniform density stockkeeping units, Int. J. Prod. Res., 2016, 54 (8), 2487-2498.

[9] EUROCOMMERCE, Retail, Wholesale in Europe, Retrieved from: https://www.eurocommerce.eu /retail-and-wholesale-in-europe.aspx, 2019.

[10] FraZelle E., World-class warehousing, material handling, McGraw-Hill, New York 2002.

[11] FrAZElle E., SHARP G., Correlated assignment strategy can improve order-picking operation, Ind. Eng., 1989, 21, 33-37. 
[12] Glock C.H., Grosse E.H., Storage policies, order picking strategies in U-shaped order-picking systems with a movable base, Int. J. Prod. Res., 2012, 50 (16), 4344-4357.

[13] Grosse E.H., Glock C.H., BALLESTER-RIPOLl R., A simulated annealing approach for the joint order batching, order picker routing problem with weight restrictions, Int. J. Oper., Quant. Manage., 2014, $20(2), 65-83$.

[14] KosińSKi W., MUNIAK R., KosińSKI W.K., A model for optimizing enterprise's inventory costs. A fuzzy approach, Oper. Res. Dec., 2013, 23 (4), 39-54.

[15] LiU C.M., Optimal storage layout, order picking for warehousing, Int. J. Oper. Res., 2004, 1 (1), 37-46.

[16] MAnzini R., Bindi F., FerRARI E., PARESCHI A., Correlated storage assignment, Iso-time mapping adopting tri-later stackers. A case study from tile industry, [In:] R. Manzini (Ed.), Warehousing in the Global Supply Chain, Springer, London 2012, 373-396.

[17] MARKETER. Global Ecommerce 2019, Retrieved from: https://www.emarketer.com/content/globalecommerce-2019, 2019.

[18] Petersen C.G., An evaluation of order picking routeing policies, Int. J. Oper. Prod. Manage., 1997, 17 (11), 1098-1111.

[19] Petersen C.G., SCHMENNER R.W., An evaluation of routing, volume-based storage policies in an order picking operation, Dec. Sci., 1999, 30 (2), 481-501.

[20] ReYes J.J.R., SOlANO-ChARRIS E.L., Montoya-TorRes J.R., The storage location assignment problem. A literature review, Int. J. Ind. Eng. Comp., 2019, 10, 199-224.

[21] RosenweIN M.B., An application of cluster analysis to the problem of locating items within a warehouse, IIE Trans., 1994, 26 (1), 101-103.

[22] Shah N.H., NAIK M., Coordinated production, ordering, shipment, pricing model for supplier-retailer inventory system under trade credit, Oper. Res. Dec., 2019, 29 (2), 55-76.

[23] TARCZYŃSKI G., Analysis of the impact of storage parameters, the size of orders on the choice of the method for routing order picking, Oper. Res. Dec., 2012, 22 (4), 105-120.

[24] Tompkins J.A., White J.A., Bozer Y.A., TANChOCo J.M.A., Facilities planning, 3rd Ed., Wiley, 2010.

[25] Van Gils T., Ramaekers K., Caris A., De Koster R.B., Designing efficient order picking systems by combining planning problems. State-of-the-art classification, review, Eur. J. Oper. Res., 2018, 267 (1), $1-15$.

[26] Wutthisirisart P., Noble J.S., Chang C.A., A two-phase heuristic for relation-based item location, Comp. Ind. Eng., 2015, 82 (2015), 94-102.

[27] Xiao J., Zheng L., Correlated storage assignment to minimize zone visits for BOM picking, Int. J. Adv. Manuf. Techn., 2012, 61 (5-8), 797-807.

[28] ZÛLJ I., Glock C.H., Grosse E.H., SCHNEIDER M., Picker routing, storage assignment strategies for precedence-constrained order picking, Comp. Ind. Eng., 2018, 123 (2018), 338-347. 